\title{
Theory of trapped polaritons in patterned microcavities
}

\author{
Pierre Lugan, ${ }^{1}$ Davide Sarchi, ${ }^{1}$ and Vincenzo Savona ${ }^{1, *}$ \\ ${ }^{1}$ Institut de Théorie des Phénomènes Physiques, Ecole Polytechnique \\ Fédérale de Lausanne (EPFL), CH-1015 Lausanne, Switzerland
}

\begin{abstract}
We consider the system of a quantum well embedded in a planar semiconductor microcavity with a shallow circular mesa patterned on top of the cavity spacer. For this system we develop the linear coupling theory of polaritons. We then compute polariton eigenstates and the corresponding optical spectrum. The theory predicts the existence of laterally confined polariton states with a discrete energy spectrum, as well as continuum states above the finite mesa potential barrier.

PACS numbers: 71.36.+c,71.35.Lk,42.65.-k
\end{abstract}

Polaritons in planar semiconductor microcavities (MCs) are quasi-particles ideally characterized by free motion in two-dimensions. The most remarkable experimental signature of free two-dimensional motion is the continuous energy-momentum dispersion curve [1, 2]. More direct evidence was recently provided by measurements of the polariton motion along the plane [3, 4], that showed ballistic propagation over hundreds of $\mu \mathrm{m}$ when polariton wave-packets were resonantly excited at large momentum.

The free particle picture, however, does not hold exactly. The structural disorder in the system breaks the translational invariance along the plane and produces localized polariton states. The polariton localization length in a GaAs-based high-quality sample was estimated in the range of a few tens of $\mu \mathrm{m}$ close to the band bottom, by measuring the momentum broadening of the dispersion curve [5], whereas recent measurements of spatially-resolved photoluminescence from a ZnSe-based sample [6] directly showed the spatial pattern of localized polariton states. Presumably, polariton localization occurs mainly due to disorder in the photonic structure. The very small polariton effective mass, as compared to the exciton mass, is indeed expected to produce motional narrowing [7] that strongly suppresses the effect of exciton disorder on the polariton states. In the $\mathrm{MC}$, on the other hand, small thickness fluctuations can produce sizeable local variations of the photon resonant energy, that are expected to affect the polariton motion.

The idea of producing a polariton trap by a local variation of the MC thickness was recently put forward [8] for engineering a "polariton quantum box", namely a photonic structure able to confine polaritons in the three spatial directions. Given the very light polariton mass, of the order of $10^{-5}$ exciton masses, the trap size required for producing a sizeable energy quantization is 300 times larger than in the case of bare excitons. Polariton quantum boxes as large as $10 \mu \mathrm{m}$ can thus be designed. Compared to quantum dots [9], these photonic structures are easier to fabricate, allowing perfect control over size, shape and positioning. Addressing a single quantum box with optical spectroscopy would also become a trivial experimental task. Another advantage with respect to extended polaritons is the reduced spectral linewidth - as is the case for quantum dots [10, 11] - due to the fact that a spatially localized state samples a homogeneous portion of the disordered MC plane. Finally, trapped polaritons still retain their quasi-bosonic character, contrarily to electron-hole states in quantum dots. Indeed, in order for the Pauli exclusion to break the bosonic behaviour, it is required that the exciton density approach its saturation value 12]. Populating a single confined state with a few polaritons still keeps the exciton density well below this limit. This quite unique situation, thanks to the discrete energy spectrum, would allow Bose-Einstein condensation of polaritons, in contrast to the ideally homogeneous two-dimensional case, for which the Hohenberg-Mermin-Wagner theorem [13] strictly forbids the occurrence of this quantum phase transition.

Let us assume a $\lambda_{c}$-thick $\mathrm{MC}$ with distributed Bragg mirrors (DBRs). Following the scheme proposed by El Daï et al. [8], in order to create a polariton trap, the thickness of the MC spacer is made slightly larger by an amount $\Delta L$ within a limited region of the MC plane that we call mesa, as sketched in Fig. 1(a). This local variation is obtained by etching the mesa pattern on top of the cavity spacer and then growing the top DBR. To an increased cavity thickness corresponds a local decrease of the resonant photon-mode energy. As the photon couples to the exciton, forming a polariton mode, the mesa acts as a polariton trap. We point out that only the MC spacer thickness is varied, while the optical thickness of the DBR layers is assumed everywhere equal to $\lambda_{c} / 4$. Therefore, within the mesa the DBRs are slightly detuned with respect to the spacer thickness. This makes fabrication easier and implies a less pronounced dependence of the local mode energy on $\Delta L$. In fact, the lowest MC resonant fequency is obtained as the solution of $\left(n_{c} / c\right)\left[\left(\omega-\omega_{c}\right) L_{c}+\left(\omega-\omega_{m}\right) L_{D B R}\right]=0$, where $L_{c}$ is the local cavity thickness, $\omega_{c}$ and $\omega_{m}$ are respectively the resonant frequency of the cavity spacer and of the DBRs, and $L_{D B R}=\left(\lambda_{c} / 2\right) n_{1} n_{2} /\left[n_{1}\left(n_{1}-n_{2}\right)\right]$ is the field penetration length in the DBRs, expressed in terms of the two DBR refraction indices $n_{1}>n_{2}$. By 

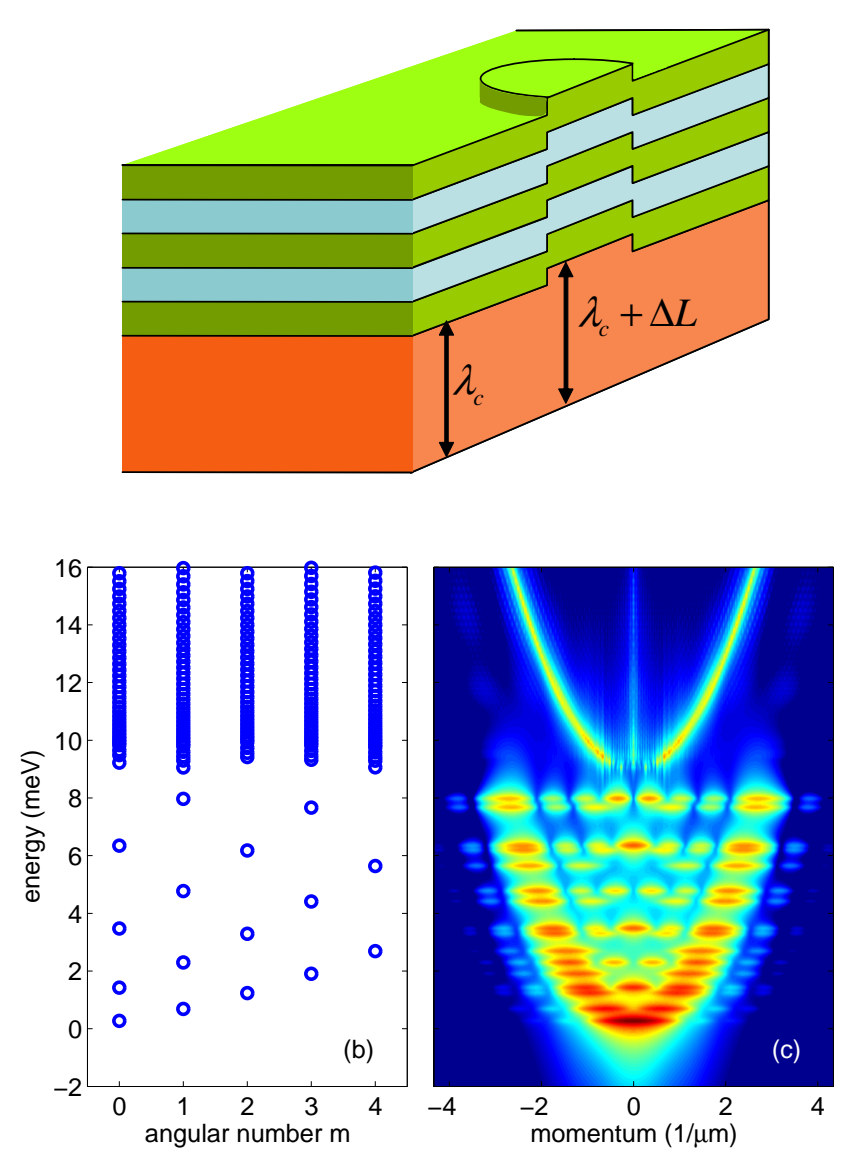

FIG. 1: (a) Sketched cross section of the mesa structure. The various lengths and the number of layers are not in scale. (b) Computed energy eigenvalues for the photon modes of a circular mesa $\left(D=8.6 \mu \mathrm{m}, \hbar \Delta \omega_{c}=-9 \mathrm{meV}\right)$. (c) Intensity plot of the photon spectral density (log scale, 4 decades from blue to red).

replacing $L_{c}=\lambda_{c}+\Delta L, \omega_{m}=\left(c / n_{c}\right) 2 \pi / \lambda_{c}$, and $\omega_{c}=$ $\left(c / n_{c}\right) 2 \pi /\left(\lambda_{c}+\Delta L\right)$, we obtain $\Delta \omega_{c}=-\omega_{m} \Delta L /\left(\lambda_{c}+\right.$ $\left.L_{D B R}\right)$. For ideal DBRs tuned to the spacer frequency, instead, $\Delta \omega_{c}=-\omega_{m} \Delta L / \lambda_{c}$. This weaker dependence on $\Delta L$ has thus the further advantage of allowing finer control over the energy offset of shallow mesas, for which a $\Delta L$ of only a few nm must be obtained in the fabrication process. For typical GaAs/AlAs DBRs, $L_{D B R} \simeq 3 \lambda_{c}$. Given $\Delta L=6 \mathrm{~nm}$ in Ref. [8], the previous expression predicts a mesa energy offset of $\hbar \Delta \omega_{c}=-9 \mathrm{meV}$, in agreement with the optical characterization of the sample.

For a shallow mesa of lateral extension larger than the optical wavelength, we can safely assume that the electromagnetic modes at in-plane position $\boldsymbol{\rho}$ are locally equivalent to those of a planar microcavity:

$$
\mathbf{E}(\mathbf{r})=\mathbf{E}(\boldsymbol{\rho}) \exp \left(i k_{z}(\boldsymbol{\rho}) z\right)
$$

where $k_{z}(\boldsymbol{\rho})$ is piecewise constant. Neglecting border ef- fects at the mesa contour, Maxwell equations give

$$
\nabla_{\rho}^{2} \mathbf{E}(\boldsymbol{\rho})+\left(\frac{\omega^{2}}{c^{2}} \epsilon_{0}-k_{z}^{2}(\boldsymbol{\rho})\right) \mathbf{E}(\boldsymbol{\rho})=0
$$

where $\epsilon_{0}$ is the spacer dielectric constant. Outside the mesa, the $\mathrm{MC}$ resonance is $k_{z}=2 \pi / \lambda_{c}$. Inside the mesa we can relate the offset $\Delta k_{z}$ to the energy offset $\Delta \omega_{c}$, as $\Delta k_{z}=\sqrt{\epsilon_{0}} \Delta \omega_{c} / c$. Eq. (2) is then solved in cylindrical coordinates, assuming a circular mesa of diameter $D$. The eigenmodes are therefore expressed as $\mathbf{E}(\boldsymbol{\rho})=U_{n m}(\rho) \exp (i m \phi)$, where $n=0,1, \ldots$ and $m=-n, \ldots, n$ are the radial and angular mode numbers respectively. The mode eigenenergies and the corresponding energy-momentum spectral function are plotted in Fig. 1 (b) and (c). A discrete energy spectrum appears, whose modes show a flat extended signature in momentum space, reflecting their spatial confinement. At energy above the $9 \mathrm{meV}$ barrier, we find a continuum of states whose energy-momentum signature practically coincides to that of a planar MC. This result already suggests that the structure is able to confine photons in the three spatial directions.

We similarly express the exciton center-of-mass wave function in terms of Bessel functions of the first kind, $\psi_{n m}(\boldsymbol{\rho})=N_{n m} J_{m}\left(\kappa_{n m} \rho\right) \exp (i m \phi)$, where $\kappa_{n m}$ are the exciton eigen-momenta and $N_{n m}$ is a normalization constant. These are the modes of a free particle, as the exciton motion is not affected by the mesa structure. By introducing Bose operators $\hat{A}_{n m}$ and $\hat{B}_{n m}$ for photon and exciton modes respectively, the linear excitonphoton Hamiltonian can be finally expressed in second quantization as

$$
\begin{aligned}
H & =\sum_{m}\left[\sum_{n} \hbar \omega_{n m}^{(p h)} \hat{A}_{n m}^{\dagger} \hat{A}_{n m}+\sum_{n} \hbar \omega_{n m}^{(e x c)} \hat{B}_{n m}^{\dagger} \hat{B}_{n m}\right. \\
& \left.+\left(\sum_{n n^{\prime}} \hbar \Omega_{n n^{\prime}}^{(m)} \hat{A}_{n m}^{\dagger} \hat{B}_{n^{\prime} m}+\text { h.c. }\right)\right]
\end{aligned}
$$

where $\omega_{m n}^{(p h)}$ and $\omega_{m n}^{(e x c)}$ are the eigenenergies of the photon and of the (free) exciton modes. As required by symmetry, the angular number $m$ is conserved in the coupling. The energies $\hbar \Omega_{n n^{\prime}}^{(m)}$ are expressed in terms of the Rabi splitting of the planar cavity $\hbar \Omega_{R}$ and of exciton-photon overlap integrals

$$
\Omega_{n n^{\prime}}^{(m)}=2 \pi \Omega_{R} \int d \rho \rho U_{n m}^{*}(\rho) N_{n^{\prime} m} J_{m}\left(\kappa_{n^{\prime} m} \rho\right) .
$$

For a planar geometry, momentum conservation implies a one-to-one coupling between exciton and photon modes. Here, on the contrary, no selection rule on the radial quantum number $n$ exists. For the numerical solution we therefore choose to retain only a finite number of cavity modes $N_{c}$ and exciton modes $N_{x}$ for each value of $m$. The resulting polariton modes are obtained by numerical diagonalization of the $\left(N_{c}+N_{x}\right) \times\left(N_{c}+N_{x}\right)$ 

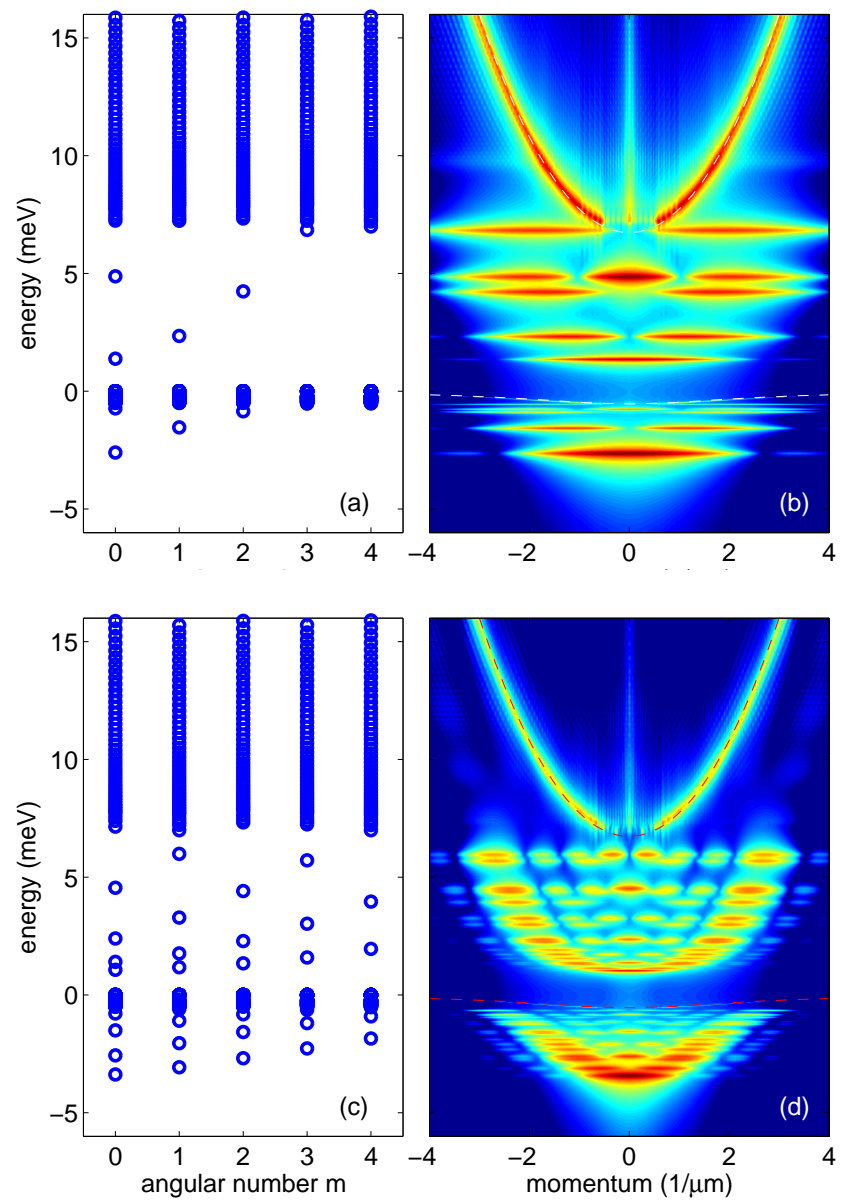

FIG. 2: (a) Computed polariton eigenvalues for $D=3.5 \mu \mathrm{m}$. (b) Intensity plot of the polariton energy-momentum spectral function for $D=3.5 \mu \mathrm{m}$ (log scale, 4 decades from blue to red). (c) and (d): same as (a) and (b) for $D=8.6 \mu \mathrm{m}$. The dashed lines indicate the bare polariton dispersion of the planar MC corresponding to the mesa barrier.

matrix obtained from the Hamiltonian (3). Polariton eigenvalues obtained for $D=3.5 \mu \mathrm{m}$ and $D=8.6 \mu \mathrm{m}$ are plotted in Fig. 2 (a) and (c) respectively. A detuning of $+6.5 \mathrm{meV}$ for the planar cavity mode with respect to the exciton was assumed, in order to bring the lowest confined photon modes in resonance with the bare exciton. The result in Fig. 22 (a) and (c) brings clear evidence of a discrete energy spectrum, followed by a continuous spectrum at higher energy, both for the lower and for the upper polariton. For the smaller mesa, only three lower polariton and four upper polariton states are present in the discrete spectrum, with energy spacings in the order of a few meV. The spectra recently measured by El Daïf et al. on mesas of comparable size show spectral features in agreement with the present model. The polariton operators obtained from the diagonalization of (3) are expressed as $\hat{P}_{n m}=\sum_{n^{\prime}}\left(X_{n m}^{n^{\prime}} \hat{B}_{n^{\prime} m}+W_{n m}^{n^{\prime}} \hat{A}_{n^{\prime} m}\right)$. Each polariton mode exhibits an angular emission pattern according to its photon component in momentum space, defined as $I_{n m}(\mathbf{k})=\left|\left\langle\mathbf{k}\left|\hat{P}_{n m}^{\dagger}\right| 0\right\rangle\right|^{2}$, that is easily computed from the model. By assuming for each mode a lorentzian energy spectrum, we can finally compute energy-momentum spectral function, as shown in Fig. 2(b) and (d). In the discrete part of the spectrum, polariton modes present a flat, broad energy-momentum signature, which corresponds to the Fourier transform of spatially confined states (not shown). The continuous part of the spectrum, on the other hand, simply corresponds to the dispersion of free two-dimensional polaritons. These are the scattering states above the finite energy barriers of the potential formed by the mesa. Correspondingly, the energy-momentum dispersion is well defined, with a negligible broadening in $\mathrm{k}$-space. We point out that the discrete modes for $D=8.6 \mu \mathrm{m}$ are less extended in momentum space than those for $D=3.5 \mu \mathrm{m}$, forming a quasi-continuum that mimicks the energy-momentum dispersion pattern of 2-D polaritons. For diameters larger than $20 \mu \mathrm{m}$, the simulations show a spectrum practically identical to that of 2-D polaritons in a MC of thickness $\lambda_{c}+\Delta L$.

In conclusion, we have developed a theoretical model of polaritons in patterned mesa structures. The model predicts a discrete energy spectrum of confined polariton states both for the upper and the lower branch, each coexisting with a continuous spectrum of extended states above the trap barrier. The confined states are extended in momentum space - a feature that should be detected in angle-resolved spectroscopy. Polariton quantum boxes are interesting in several respects. The quantum confinement is produced in a way that is not as invasive as in micropillars [14], which seem to considerably reduce the quality factor of the photonic structure, presumably by opening lateral emission channels. Confined polaritons represent a quite unique system of quantum-confined interacting bosons. They can undergo parametric scattering processes - due to the mutual Coulomb interaction - that have recently been exploited for producing nonclassical states of polaritons [15]. In quantum boxes, momentum conservation no longer holds and parametric processes between quantum confined states become allowed. This might be exploited for designing a quantum optical device. Recently, rigurous proof was given, that a discrete energy spectrum allows Bose-Einstein condensation [16], otherwise impossible in two-dimensions [13]. Polariton quantum boxes, could therefore be the ideal system for observing the long sought Bose-Einstein condensation of polaritons.

We are grateful to B. Deveaud, W. Langbein and R. Zimmermann for the fruitful collaborations. Financial support by the Swiss National Science Foundation (project No. 620-066060) is also acknowledged. 
* vincenzo.savona@epfl.ch

[1] R. Houdre et al., Physical Review Letters 73, 2043 (1994).

[2] W. Langbein, Journal of Physics-Condensed Matter 16, S3645 (2004).

[3] W. Langbein, Microcavity polariton dynamics in real and reciprocal space, in Proceedings of the 26th International Conference on the Physics of Semiconductors, edited by A. R. Long and J. H. Davies, Institute of Physics Publishing, 2002.

[4] T. Freixanet, B. Sermage, A. Tiberj, and R. Planel, Physical Review B 61, 7233 (2000).

[5] W. Langbein and J. M. Hvam, Phys. Rev. Lett. 88, 47401 (2002).

[6] M. Richard et al., Phys. Rev. B 72, 201301(R) (2005).

[7] D. M. Whittaker, Physical Review Letters 80, 4791
(1998)

[8] O. E. Daf et al., Applied Physics Letters 88, 061105 (2006).

[9] D. Bimberg, M. Grundmann, and N. N. Ledentsov, Quantum Dot Heterostructures (John Wiley \& Sons, 1999).

[10] D. Gammon, E. S. Snow, B. V. Shanabrook, D. S. Katzer, and D. Park, Science 273, 87 (1996).

[11] P. Borri et al., Phys. Rev. Lett. 87, 157401 (2001).

[12] S. Schmitt-Rink, D. S. Chemla, and D. A. B. Miller, Physical Review B 32, 6601 (1985).

[13] P. C. Hohenberg, Physical Review 158, 383 (1967).

[14] G. Dasbach, M. Schwab, M. Bayer, and A. Forchel, Physical Review B 64, 201309(R) (2001).

[15] S. Savasta, O. D. Stefano, V. Savona, and W. Langbein, Physical Review Letters 94, 246401 (2005).

[16] J. Lauwers, A. Verbeure, and V. A. Zagrebnov, Journal of Physics a-Mathematical and General 36, L169 (2003). 